\title{
Making Sense of Aljazeera's Televised News: A Schema Theory Perspective
}

\author{
NAjib Slimani \\ Correspondence: Najib Slimani, Ecole Nationale de Commerce et de Gestion, Avenue Allal El Fassi B.P. 3748, \\ Amerchich, Marrakech, Morocco.
}

Received: December 30, 2015 Accepted: January 11, 2016 Online Published: March 1, 2016

doi:10.11114/smc.v4i1.1427

URL: http://dx.doi.org/10.11114/smc.v4i1.1427

\begin{abstract}
Realizing that there exists a definite dearth of information on how English televised news discourse is processed by non-native viewers, I seek to examine how Moroccan viewers make sense of Aljazeera 24-hour English (henceforth Aljazeera) news discourse in terms of comprehension and recall. In light of schema theory research (Carrell, 1981; 1983; Hoijer, 1987; 1989; 1991; Kintsch, 1989), I designed pre-, while- and post-viewing tasks to explore the extent to which Moroccan viewers' linguistic and extra-linguistic prior knowledge mediate their processing of Aljazeera's televised news content. This study reveals that viewer-bound parameters, namely relevant-topic knowledge, linguistic literacy and familiarity with televised news formats, truly mold the way non-natives process authentic English news discourse.
\end{abstract}

Keywords: Aljazeera news, background knowledge, moroccan viewers, schema theory, interpretation

\section{Introduction}

This study fits within the reception analysis model of media discourse. It investigates parameters linked to the position of audiences as media recipients. Historically, the fact that audiences can be potential meaning-makers has been recognized by several researchers within and across disciplines, namely media and discourse comprehension (Bower, 1973; Cardwell, 2005; Hamilton, 2004; Kintsch, 1977; 1978; 1998; Leighly, 2004; Morris, 2004, Starr, 2004; van Dijk, 1999). Against this background, the present study seeks to examine how Moroccan audiences make sense of televised news discourse. By design, televised discourse encompasses a wide variety of linguistic and non-linguistic constituents which require active, cognitive processing. As such, audiences are called for to be disposed to cater for news content before-, while- and after entering a news situation.

In this work, I support the view that the existence of utterly passive media audiences is a sheer fallacy-a postulate that has been supported by proponents of the active audience media theories. I rather maintain that audiences' position in the mass communication process is of central importance. Taking into account the overall structure of contemporary media news content which encompasses multifarious audiovisual implications, it would be foolhardy to continue to believe that media audiences unquestionably sponge in media messages. A quick survey of prominent televised news channels' agendas would be enough to build up a conviction that viewers' opinions about salient daily issues are accounted for, if not fore-grounded. It is particularly important to specify that viewers' opinions, attitudes and worldviews are not shaped from a vacuum. Television viewers are equipped with a bulk of background knowledge which mediates and conditions their attribution of specific interpretations to televised news content.

\section{Background Knowledge: A Schema Theory-bound Concept}

In the literature, background knowledge is referred to as 'knowledge scripts or slots' which enable a person to make sense of, retrieve or remember information, be they print, audio or audio-visual (den Uyl \& van Ostenoop, 1980). According to these researchers, background or prior knowledge experiences are of key importance not only in decoding and organizing input information but also in remembering and retrieving specific information already stored in memory.

Noticeably, scholars have been challenged by attempts to come up with a concise, clear-cut delimitation of a person's background knowledge. As a concept, background knowledge incorporates a wide range of experiential patterns and information, such as linguistic knowledge, cultural convictions, familiarity with the textual and/or audio-visual format of media content, and so on and so forth.

There seems to be a broad set of cross-disciplinary research related to the construction and reconstruction processes of 
coping with information. In this respect, the use of prior knowledge in processing new information is an issue which finds its roots in previous research and studies undertaken in language learning. As a case in point, Carrell (1981; 1983), a pioneer of schema theory in language learning literature, provides detailed experimental data about the pivotal significance of learners' prior knowledge in the cognitive processing of reading comprehension materials in the pre-reading, while-reading and post-reading stages.

Being aware that television viewers may engage in cognitive processes akin to language learners, some media researchers (Reeves and Thorson, 1986; Pezdek, et al. 1987; Eckhardt et al. 1991; Findahl and Hoijer, 1985; Hoijer, 1989) accentuate the role of background knowledge as a schematic variable which conditions and mediates viewers' processing of media content. This research trend not only gives strong support to the basic premises of the schema theory, but it also provides tangible empirical evidence that television viewers may engage in processes and strategies similar to language learners in the comprehension and recall processes of televised information.

\section{Schemata and Television Comprehension}

A survey of the literature on television content processing clearly indicates that the use of individual schemata to make sense of televised programming is a widely acknowledged fact. Levy and Windahl (1985), for instance, show that active processing of television content necessitates deep reflection through an ongoing, active use of one's prior knowledge structures. Hoijer (1989: 197) shares the same viewpoint empirically proving that a viewer who is endowed with topic-relevant previous knowledge is basically an active discourse processor unlike a viewer who lacks this privilege. The latter is a more passive discourse processor for he/she takes everything for granted instead of comparing and contrasting televised content with his/her pre-acquired knowledge structures.

The literature on media reception and interpretation has struggled to determine the ramified functions of schemata or prior knowledge in the comprehension of televised content. In this respect, Findahl and Hoijer (1985: 379) emphasize the instrumentality of viewers' background knowledge in interpreting and recalling televised information. Using fieldwork research, these researchers seem to have developed a hard-to-dislodge conviction that viewers with high verbal ability and rich prior knowledge are at a great advantage when it comes to processing televised content compared to viewers with less verbal ability and reportorial knowledge. In the context of this experiment, verbal ability refers to the capacity to speak, use and understand a specific language.

It seems that the significance of schemata or prior knowledge in processing input information is recognized by scholars within and across disciplines; mainly language studies and media studies. The notion of schemata gains even more tangible momentum in audio-visual discourse processing. The latter discourse is endowed with some discourse properties, namely the use of 'moving pictures' or visuals, which set it apart from prosaic discourse where the scope of factual comprehension and recall is mostly conditioned by the 'written word'. The question remains, to what extent does non-native viewers' prior knowledge mediate their comprehension and recall of authentic English news content?

In this study, I examine the significance of content-bound and format-related background knowledge in processing English news content by non-native English speakers. In specific terms, I investigate how viewers' background knowledge, including familiarity with news format, linguistic literacy, cultural consciousness and parallel parameters, may determine Moroccan viewers' processing of Aljazeera English (henceforth Aljazeera) news programming in the pre- and while-viewing stages. In a parallel research axis, I focus on how Moroccan university or college students use visuals in the post-viewing recall of the gist of Aljazeera's news stories' content, in particular who says, what, to whom, with what effect and related thematic and vocabulary-specific parameters.

Eventually, I choose an audience-oriented research axis whereby I intend to scratch on non-native viewers' interpretation and recall mechanisms of English televised news discourse. In this regard, the consideration of audience-related parameters is an outspoken claim of several contemporary media discourse researchers who assume that audience-oriented analyses as a first step away from text-focused analyses may unpack new findings about the working of media discourse (Schiffren et al. 2003).

The innovativeness of this study lies in the fact that it is one of the first of its kind to engage Moroccan audiences with a view to examine the afore-mentioned research parameters. This study may unveil new factors that determine how Moroccan viewers make sense of televised news discourse. It may also reveal that western theories of media research may not be appropriate or need to be restructured in a way which truly captures non-western thinking.

\section{Research Method}

In the present investigation, I opt for the focus group as a handy way to obtain what might be hard-to-obtain feedback. The choice of the focus group to investigate the way audiences process and make sense of televised news content could be justified by two main reasons: First, there seems to be a scholarly tendency to believe that the focus group is probably one of the most appropriate approaches to analyze audiences' response to media content. Stokes (2003: 148) 
states that "if you want to find out why people believe what they do, understand more of the nuanced reasons behind their answers or question them about their opinions of particular media texts, focus groups are probably the best approach." Second, the focus group technique entails working with a limited, homogeneous number of respondents. In this regard, I had access to potential subjects, namely young Moroccan university students doing advanced English studies and having the required profile for a comprehensive investigation of different aspects of the issue at hand. Here, it is important to specify that the feedback of the focus group was considered at the collective rather than the individual level throughout the experiment. The comparison is undertaken among the sub-groups within the focus group participants.

My analysis of pertinent research variables is researcher-related. A researcher-bound method would help me to observe and analyze the contours and processes the participants use to understand and interpret the discourse structures of the broadcasts under investigation. In fact, I undertake a nominal, rather than numerical, analysis of the participants' cognitive processes in the three phases of the viewing experience i.e. before they watch, while they watch and after they watch the televised news stories.

\subsection{Research Population and Sample}

With respect to the selected spectrum of viewers, I targeted participants with the following profile: educated, English-literate and media-oriented. Moroccan university students doing advanced English-related studies at the university level turned out to be an appropriate option. In fact, the participants' linguistic literacy constituted a guarantee for their actual possession of the qualities required for providing high-validity feedback in the context of this investigation. The option of Moroccan respondents could be justified by the realization that there is a scarcity of academic research on how Moroccan viewers use and process authentic English news content.

Before recruiting participants, I visited advanced and proficiency class students, talked to them about my research project and invited them to fill in a release form as a testimony to their willingness to take part in the project. Eventually, I ended up with eighteen active participants. Most of the students who did not want to participate confessed that because of exam preparation constraints they could not join in. Out of the 18 participants, twelve are female and 6 are male, and their age ranged between 17 and 23. In addition to their presumably broad range of background knowledge about multiple news issues, the participants are language people, and this makes it easy for them to process the news material in English without any need for translation.

\subsection{Data Collection and Testing Measures}

To obtain data for this research project, I chose Aljazeera news channel. The choice of Aljazeera, among a plethora of news stations, could be justified by the results I obtained from an earlier study centered on the effects of Aljazeera on Moroccan viewership. The study revealed that Aljazeera is a viable viewing alternative for English-literate Moroccans. Thus, I retrieved two news stories from the archives of Aljazeera's online database. The stories cover the following issues:

Ending violence against women: A Jordanian organization offers shelter

Illegal Immigration: The town of Cittadella in Italy as a showcase

The storylines of these broadcasts include the discourse schema of a standard news story. Each story was presented in the form of a clip made of four major constituents: (1) summary/introduction where the major headlines of the lead stories were outlined, (2) episode/events where previous information was matched with current events and further explanations were provided, (3) consequences where the prospective future effects of the current events were spotlighted, and (4) comments/reactions where guest speakers, specialists and sometimes ordinary people were invited to voice their opinions about the broadcast events.

Basically, the testing measures adopted in this study consisted of three distinct stages/tasks: A pre-viewing stage task whereby participants' background knowledge was browsed, a while-viewing stage task whereby participants' actual cognitive abilities were tested, and a post-viewing stage task whereby participants' recall capacities were assessed. Accordingly, in the pre-viewing task participants were asked general-topic questions, such as web or diagram completion and/or general topic-related questions, to evaluate their degree of familiarity with the broadcasts' contents.

In the while viewing stage, topic-specific questions were designed to measure participants' understanding of major as well as minor events in each news story. A master list of five true/false questions was designed to elicit participants' comprehension and interpretation of each storyline. The post-viewing task, which was assigned two days after the accomplishment of the while-viewing task, targeted assessing the participants' recall capacities of the contents of the two storylines. At this stage, participants were asked to watch each news story in the visual mode only (without verbal content) and fill in a recall chart which basically summarizes the overall content of the story. Specifically, participants were asked to recollect the event, place, characters, cause and consequence of each storyline. Besides, participants were 
asked to provide personal interpretations of each storyline and, finally, to recall key vocabulary items mentioned in each news report. To evaluate participants' comprehension and recall abilities, I adopted a 0-5 point scale for each set of questions. Students get a score of five if they came up with relevant responses, four if the evaluation criteria were not fully met, three if the answers were less relevant, etc. In the results and discussion section, I present the participants' responses and proceed with a nominal, non-numerical discussion of their implications.

\section{Findings}

The theme of the first newscast was violence against women- an issue which has always been a real taboo in the Arab world. Currently, this issue is gaining momentum as a top-priority concern for people from different walks of life both within and across Arab countries. In Morocco, for example, women who are victims of violence seem to benefit from an unprecedented nationwide support from the organizations of the civic society. The news broadcast under study presented cases of Arab women victims of violence and the efforts made by a Jordanian organization to bring relief for those pity-arousing cases.

\subsection{The Pre-viewing Experiment}

In the pre-viewing task 1, I brainstormed the participants' general-topic knowledge by asking them to provide answers for two opinion questions:

What are the characteristics of violence against women?

How can we put an end to the countless cases of violence against women?

In general, the group members came up with brief, but accurate, answers to the first question. They specified that violence against women could be sexual, physical or psychological. In my viewpoint, these three terms may be considered as umbrella terms which actually host multiple forms of violence against women starting from verbal harassment to brutalizing them in any other ways.

The responses of the participants to question two clearly indicated their full awareness that solving this issue was a real, social challenge. Some of the key terms which figured in the group members' responses were full of significance: social education, family sensitization, governmental organizations as well as non-governmental organizations contributions, etc. Obviously, the participants seemed to grasp some of the general traits and ramifications of the issue at hand.

The second pre-viewing task consisted of getting the participants to conduct an open, group discussion of the theme of violence against women. While being a genuine opportunity for the participants to exchange their opinions about this issue, the group discussion was a live and experimental outlet to promote researcher-bound analysis of the group's views and opinions. According to the discussion, there was a unanimous agreement among the participants that harassing women in any way had to be considered as a crime that should incur severe legal punishment in tune with the seriousness of the harassment. Some participants mentioned that the US is to be taken as a model country given the zero-tolerance laws implemented in this respect. The group members also affirmed that violence against women could be culture-bound, and that there were cultures where this type of violence was widely tolerated. With regard to Morocco, the participants concurred that civic associations and organizations were making strenuous efforts to sensitize people about the dangers of this phenomenon and to provide social/psychological shelter for women in difficult situations. The group members reaffirmed their deep-seated conviction that resolving this issue was a long process requiring utmost collective efforts.

The group members showed a remarkable ability to convey ideas about their understanding of different facets of the theme of violence against women. I need to specify, though, that the participants' prior knowledge was culture-specific, topic-specific and also vocabulary-specific. In fact, the group members expressed their awareness that there were some non-western cultures where this kind of violence was a matter of fact and where a lot of work had yet to be done. The topic-specific awareness of the participants could be deduced from their insistence on the pivotal role of governmental and non-governmental organizations to end this violence. Finally, the richness of the group members' vocabulary-specific repertory was evident in their ability to provide different specific terms related to this issue.

The pre-viewing activities relevant to broadcast story 2 consisted of two written and two oral tasks. For the first written task, the participants were asked to fill in a chart where they had to specify three pull factors and three push factors related to illegal immigration. We explained that 'push factors' were basically negative factors which pushed immigrants to flee their countries of origin in any illegal way possible. We also made clear to the participants that 'pull factors' were positive aspects of the host countries' social system which tempted the candidates for illegal immigration. The group members, indeed, listed a variety of push/pull factors, which gave the impression that they had in-depth background information about this prevalent phenomenon in their home country- Morocco. Examples of push factors cited by the participants were: poverty, unemployment, political unrest, social insecurity, etc. Participants also cited pertinent pull factors, such as good living conditions, job opportunities, social security, human rights, etc. 
In the second written task, the group was asked to specify and comment on some of the potential difficulties illegal immigrants were bound to encounter once in the host countries. Responses to this question also showed that the group members were knowledgeable enough about the surrounding features of illegal immigration. The participants shared the point that candidates for illegal immigration would definitely undergo dire social and psychological consequences because they would be deprived of the basics of decent living conditions, decent accommodation, job opportunities, social security, etc. Lastly, the participants issued comments which showed that dreams of illegal immigration were nothing more than illusive rainbows.

The pre-viewing activities consisted of pair and group work tasks in which the participants were invited to speak out their opinions about different facets of the illegal immigration phenomenon. While monitoring the pair-work activity, we noted the heated discussions which took place among the pair-workers. Similarly, in the group work activity, the participants ignited an insightful debate in which almost all the features of this phenomenon were unveiled. They specified that illegal immigration was a risky endeavor which might cost immigrants their lives. They exemplified the miserable living conditions of illegal immigrants. There was a consensus that illegal immigrants were often considered as social outcasts. Importantly, the participants emphasized the urgent need to work out solutions for illegal immigrants in the host countries and, most importantly, in the home countries. Thus, during all the stages of the pre-viewing activities, I noticed that the participants had diversified background knowledge about the phenomenon of illegal immigration and related issues. This did not come as a surprise because the group members confirmed their utmost concern for this issue which hooked public attention both within and across nations.

In the pre-viewing phase, I drew upon educational research techniques to tap the participants' existing background knowledge (Hess, 1991). Pre-viewing tasks were assigned to increase the participants' curiosity, stir their imagination, foster their motivation and give them a sense of purpose for watching the broadcasts. The techniques used, which were not all-inclusive, ranged from creating a semantic map through answering topic-related questions to performing pair and group oral tasks. Employing their experiential, world knowledge as well as their imagination and intelligence, the participants exhibited skills which approximated those of mature discourse processors. In a word, the participants set a tangible example of viewers who did not enter the televised news situation as 'blank sheets'. Their prior knowledge was so rich and diverse that they could visualize insightful responses to the pre-viewing tasks. What remains yet to be explored is the extent to which the participants' prior knowledge would mediate their processing of the while- and post-viewing questions.

\subsection{The While-viewing Experiment}

The main theme of broadcast story 1 was violence against women in the Arab world. The news story was an interview between Aljazeera's news reporter and some women's rights activists in the Arab world. The case of violence against women in Jordan and the role of non-governmental organizations constituted a principal focus point of this news report. In light of this background, I designed true/false questions which would elicit the participants' understanding of the main events of the news report, their actual consequences and their probable future turnouts:

The name of the organization is the Lebanese Women's Shelter.

The shelter can hold up to 25 women.

Women are better informed about available services now.

Hibaaq Osman thinks the UN initiative can help women.

Violence against women is no more a taboo topic in Arab countries.

The group members' response to question 1 tangibly showed that they made good sense of the organization in questionthe Jordanian Women's Union Shelter. In fact, a good part of the report was centered on the role of this union in the struggle to restore Jordanian women's rights and dignity.

In question 2, we elicited an important detail in the storyline- the shelter's hosting capacity. The answers to this question indicated that the participants got confused by numerous numerical figures which were mentioned in the report. Some participants reported that the actual number of abused women the center could hold was thousands, but this was the number of cases the shelter had received since 1999. The actual hosting capacity of the center was actually twenty-five.

Question 3 targeted the group's understanding of the results of the union's strenuous efforts to make Jordanian women aware of its existence as an organization which actually helped Jordanian women in distress. Overall, the participants made the right answer choices and elaborated on their answers in fully comprehensible ways. They used report-based justifications:

Women were more aware of the social services the shelter could provide.

The existence of the shelter was not a secret anymore for Jordanian women. 
Jordanian women knew that the shelter could solve their problems in different ways.

Question 4 elicited the participants' grasp of the attitude of Hibaaq Osman, a representative of the organization 'Karama', towards the UN initiative to end violence against women. The participants unanimously reported that she was supportive of the initiative, but she stressed that no grass-roots changes could be made through that initiative in the Arab world. The last question was about Hibaaq Osman's viewpoint about the issue of violence against women in the Arab world- is it a taboo or not anymore? The participants' responses to this question proved their ability to make sense of the fact that it was a taboo for reasons that Hibaaq Osman pointed out:

It is a taboo for cultural reasons.

It is still a family affair.

The main theme of broadcast story 2 was about the situation of an illegal immigrant couple living in an Italian town, Cittadella. The mayor of the city enacted a law which aimed at expelling illegal immigrants. Aljazeera's reporter shed light on the repercussions of the new law on the living conditions of the couple. The true/false questions tuned with the discursive structure of the report which was molded in a way to show the injustice of the new law:

EU law was used to dictate a new law of immigration.

The people concerned by the new law are happy and satisfied.

Yuri and Victoria are examples of immigrants who benefit from the new law.

The region's economy does not depend on illegal immigrants.

Only one Italian town has decided to adopt the new law.

The first question was about the main theme of the newscast, which was using EU law to dictate a new law of immigration. The participants accessed this information quite easily. They provided report-based justifications which proved that the mayor of the town relied on EU legislation to pass the new law:

The mayor used EU law to dictate what type of foreigners can live here.

Question 2 was about the psychological repercussions of the new law upon illegal immigrants in the town of Cittadella. Once again, the participants retrieved the right information and justified with story-based statements like:

The people affected by the law aren't happy.

I guess that the participants' ability to have quick access to this information could be ascribed to the discursive structure of the newscast. From the outset of the report, the reporter emphatically reported the immigrants' utmost dissatisfaction with the new status quo.

The third question milled around the group's ability to describe the living situation of Yuri and Victoria as a case example of immigrants who were concerned by the new law. The group reported the falsehood of the statement which indicated that the couple benefited from the new law. The reaction of Yuri and Victoria to the new law was fully commented upon by Aljazeera's news reporter, and the group members accessed the right information:

They don't feel welcome.

The new law shuts people like them out.

They are frustrated at the way they are being treated.

Providing such justifications clearly indicated that the participants inferred the unfairness of the new law for illegal immigrants, Yuri and Victoria as cases in point.

The penultimate question was about the contribution of illegal immigrants to local economy. The reporter expressed her support for illegal immigrants and stressed the opportunistic attitude of the mayor's ordinance- a piece of information which the participants grasped through their positive descriptions of illegal immigrants:

They provided cheap labor.

The region became one of the wealthiest in Europe thanks to illegal immigrants.

The region's economy absolutely relied on illegal immigrants.

In the last question, I elicited the participants' grasp of the probable, future development of the story's main eventenacting the new law across Italy. By the end of the report, the reporter mentioned that a considerable number of towns, in the region of Cittadella and across Italy, were willing to adopt the new law. The participants retrieved this information and provided newscast-based statements to justify their views:

Two hundred towns in the region have decided to take similar anti-immigration measures. 
Dozens of towns across Italy look forward to adopting the new law.

In summary, the participants' responses to the true/false statements showed their ability to make sense of the central points of all the news stories. Although some participants did not manage to grasp and/or justify very few questions, it remained obvious that they were clear about the overall discourse structure of each news story. This asset may be ascribed to their topic-specific knowledge, to their heightened level of perceptive listening/watching and also to their distinguished levels of linguistic literacy. These background knowledge factors definitely mediated the participants' comprehension and interpretation of the storylines in the while-viewing phase. Now, let us examine whether the participants would exploit their background knowledge to handle the post-viewing questions with the same alertness.

\subsection{The Post-viewing Experiment}

For sure, processing televised news information is a process which starts before getting exposed to news bulletins, during exposure and, most importantly, after exposure. In the post-viewing stage, viewers' processing involves numerous parameters, namely recalling the content of the news bulletins and developing personal, interpretive responses to them. Within this realm, the post-viewing task took place two days after because I targeted the participants short-term recall capacities. In this activity, images are used to brainstorm and activate participants' background knowledge. I asked the participants to fill in a researcher-designed information chart which summed up the major discursive aspects of each news bulletin in terms of content, personal interpretation and story-related vocabulary.

In the recall questions $1,2,3$, and 7, we aimed at eliciting the group's ability to recall the actual events leading to each news story, to specify the angles from which the story was covered, to list some key phrases and sentences summarizing the storyline as well as recapture key lexical items in each story. In the personal interpretation questions 4 , 5, and 6 , we tested the participants' ability to figure out a possible headline for each newscast, to find out possible angles from which the stories' issues could be covered and finally to state whether or not the stories strengthened the participants' prior knowledge about the news issues.

The participants' displayed a distinguished ability not only to recall the contents of the newscasts but also to go beyond that by raising thought-provoking points which pertain to the news issues. For broadcast story 1, the participants generally showed high recall competence of the outstanding discourse parameters of the story. Specifically, they recalled that the story was based on social cases of women victims of violence in Jordan. The participants also specified the attitudes of the guest speakers as women rights' activists and listed some vocabulary words which summarized the plot of the news report. On a related level, I found that the group's interpretation faculties were rich and thoughtful for they suggested different new angles from which the story could be covered, specified new information that the reporter could have added and suggested attractive headlines for the story.

However, what applies to the first broadcast does not exactingly apply to the second broadcast. I found that the participants were able to recall the overall discourse schemata of the newscast, but they generally did not manage to recall some recurrent information in the newscast. For example, some participants could not recall who issued the new law of immigration and where this law was enacted. Some participants failed to recall the names of the illegal immigrant couple whose situation was amply covered in the newscast. Although the participants missed some factual information deeming them less central to the storylines, they exhibited insightful interpretation capacities of the newscast's main issue and came up with various pertinent headlines for the story. Regardless of this discrepancy, the role of visuals as a 'meaning-potential' component which enhanced the viewers' recall and interpretation faculties was an experimental outcome of the post-viewing tasks.

All the viewing phases considered, I found that the ability to comprehend, interpret and recall televised news content was mediated by several factors, namely the richness of the general-topic knowledge and linguistic literacy. Since individuals differ in these skills, there is a reason to believe that each individual member should process information at his/her own pace and capacity. Still, the collective focus group feedback in this study unpacked that the above factors indeed mediated Moroccan students' processing of televised news discourse in the pre-, during-, and after-viewing phases.

\section{Conclusion}

This study was an attempt to provide deeper insights into some of the ways Moroccan recipients make sense of authentic English news discourse. I sought to examine some facets of televised news discourse reception in light of a recipient-oriented education theory i. e. the schema theory. The choice of this theoretical perspective was justified by a personal academic conviction that testing this education-bound theory in a non-western media context might yield eye-opening results relating to media discourse reception among non-natives. Specifically, I examined how Moroccan university students made sense of Aljazeera's news programming in the pre-, while-, and post-viewing stages. In the context of this study interpreting and recalling inextricably relate to the process of making sense of televised news discourse. 
I started from a well-known presumption in educational research that language learners, especially in reading activities, are usually endowed with a repertory of prior knowledge which needs to be activated in the pre-reading stage and exploited in the while- and post-reading stages to make better sense of the reading material. Faithful to this perspective, I examined the significance of viewers' background knowledge in making sense of televised news discourse.

Because background knowledge is, by definition, a multi-faceted component, I specified that what I meant by background knowledge in the context of this study was the following: personal prior topic knowledge, familiarity with televised news format, personal linguistic literacy and information cognitive processing capacities. To test the significance of these parameters on televised discourse comprehension and recall, I designed pre-, while-, and post-viewing tasks. This study revealed the substantial significance of background knowledge in making sense of authentic English news content.

The limitation of this study could be ascribed to the research technique, which is the focus group. In fact, the focus group entails working with a limited number of respondents, which makes it hazardous to generalize its findings over a large spectrum of the Moroccan population. Regardless of this limitation, the credit of this study is that it zooms on an important research paradigm that has hardly ever been investigated by media researchers, that is non-natives processing of authentic English televised news programming.

Future research endeavors related to this paradigm would definitely dissipate haziness around this issue. It could be possible to examine the same research parameters on a bigger, diversified sample of the Moroccan populace. It could also be worthwhile to scrutinize the significance of other factors, such as gender affiliation, level of education and/or professional status, in processing televised news content. Another research axis might focus on the 'proximity' factor as a variable in making sense of televised news content. That is, would non-native viewers process news issues with which they are familiar in the same way as unfamiliar news stories? Accordingly, this study may be the start-up of a journey on a yet-to-be explored research path.

\section{References}

Bower, T. (1973). Television and the public. New York: Holt, Rinehart \& Winston.

Cardwell, M. (2005). Media bias and media markets. Paper read at the Annual Meeting of The American Political Science Association, Washington D. C.

Carrell, P. L. (1981). Culture-specific schemata in L2 comprehension, 14-39. Orem, R. A., Haskell, J. F. eds. Selected papers from the ninth Illinois TESOL conference. Chicago, Illinois.

Carrell, P. L. (1983). Three components of background knowledge in reading comprehension. Language learning. 34, 87-112. http://dx.doi.org/10.1111/j.1467-1770.1984.tb01005.x

Den Uyl, M., \& Oostenoop, H. (1980). The use of scripts in text comprehension. Poetics, 9, 275-294. http://dx.doi.org/10.1016/0304-422X(80)90023-6

Dijk, T. A. (1999). Context models in discourse processing. pp. 123-148. In: van Oostendorp, H., Goldman S. R. eds. The construction of mental representations during reading. Mahwah: NJ, Erlbaum.

Eckhardt, B., Wood, M., \& Jacobwitz, R. (1991). Verbal ability and prior knowledge: Contributions to adults' comprehension of television. Communication Research, 18, 636-649. http://dx.doi.org/10.1177/009365091018005004

Findahl, O., \& Hoijer, B. (1985). Some characteristics of news memory and comprehension. Journal of Broadcasting and Electronic Media. 29, 379-396. http://dx.doi.org/10.1080/08838158509386594

Hamilton, J. (2004). The market and the media. pp. 31-67. In: Overholser, G., Jamieson, K. H. eds. The press. Oxford: Oxford University Press.

Hess, N. (1991). Headstarts: One hundred original pre-text activities. New York: Longman.

Hoijer B. (1989). Television-evoked thoughts and their relation to comprehension. Communication Research, 16, 179-203. http://dx.doi.org/10.1177/009365089016002002

Hoijer, B. (1987). Natural science in television: Comprehension of and responses to a programme. Stockholm: Swedish Broadcasting Corporation.

Hoijer, B. (1991). Reception reconsidered from comprehension perspectives. Paper delivered at the 11th Nordic Conference for Mass Communication Research, Trondheim.

Kintsch, W. (1977). On comprehending stories. Just, M. A., Carpenter, P. A. eds. Cognitive processes. Hillsdale, NJ: Lawrence Erlbaum Associates. 
Kintsch, W. (1978). Comprehension and memory of text. In: Estes, W. K. ed. Handbook of learning and cognitive processes. Hillsdale, NJ: Lawrence Erlbaum.

Kintsch, W. (1998). Comprehension: A paradigm for cognition. Cambridge: Cambridge University Press.

Leighly, J. E. (2004). Mass media and politics: A social science perspective. 33-52. Cigler, A. J., ed. New directions in political behavior. Boston: Houghton Mifflin Company.

Levy, M. R., \& Windahl, S. (1985). The concept of audiences activity. pp. 109-122. In: Rosengren, K. E., Wenner, L. A., Palmgreen, P. eds. Media gratifications research: Current perspectives. Beverly Hills, CA: Sage.

Morris, J. S. (2004). The Fox news factor: Predicting americans' exposure to cable and nightly network news. Paper read at the Annual Meeting of the Midwest Political Association, Chicago.

Pezdek, K., Simon, S., Stoeckert, J., \& Kiely, J. (1987). Individual difference in television comprehension. Memory and Cognition, 15, 328-435. http://dx.doi.org/10.3758/BF03197732

Reeves, B., \& Thorson, E. (1986). Watching television: Experiments on the viewing process. Communication Research, 13, 343-361. http://dx.doi.org/10.1177/009365086013003004

Schiffrin, D., Tannen, D., \& Heidi, E. H., eds. (2003). The handbook of discourse analysis. Oxford: Blackwell. http://dx.doi.org/10.1111/b.9780631205968.2003.x

Starr, P. (2004). The creation of the media: Political origins of modern communication. New York: Basic Books.

Stokes, J. (2003). How to do media and cultural studies. London: Sage Publications Ltd.

\section{(cc) BY}

This work is licensed under a Creative Commons Attribution 3.0 License. 